\title{
THE ESTIMATION OF DISSOLVED OXYGEN IN WATER.
}

By A. Dupre, Pн. D., F.R.S., \&c.

(Read at the Meeting on 10th June, 1885.)

Up to the year 1872, the estimation of the oxygen dissolved in water had to be accomplished by means of a somewhat troublesome and time-taking process, in consequence of which this feature of water analysis was very greatly neglected. In that year, however, P. Schützenberger and Ch. Rister described a process in which they made use of the remarkable reducing power of an acid discovered by Schützenberger in 1869, and called by him hydrosulphurous acid, now known as hyposulphurous acid or dithionous acid. By the help of this acid, or rather of its sodium salt, the estimation of the oxygen dissolved in water becomes at once easy and rapid, and $I$ have no doubt that everyone once familiar with the process, will very frequently employ it. It is this process, with some modifications introduced by myself and others, which I propose to bring before you this evening.

In the year 1881, while engaged in an extensive series of analyses of Thames water, my attention was, among other features, directed to the varying amounts of dissolved oxygen contained in the river water at different parts of the river, and after trying various processes, I finally adopted the one to be described. The chief difference between it and the original process consists in making use of fully ærated water for standardizing the hyposulphite solution, in place of the ammoniacal sulphate of copper, used by Schützenberger and Rister.

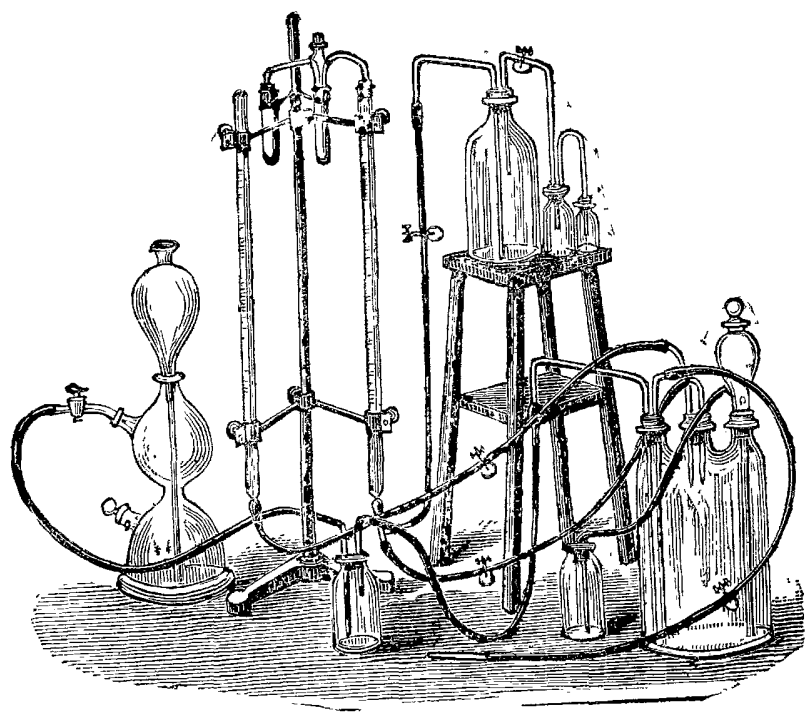

'The apparatus employed, which, somewhat complicated at first sight, works very easily, is as follows :-One neck of a three-necked. Woulf's bottle, of about one quart capacity, is connected, by means of a doubly-perforated india-rubber stopper, with two burettes for containing indigo and hyposulphite solutions respectively. The hyposulphite burette is connected, at its lower end, in the usual manner, with a supply bottle holding the hyposulphite solution, and both burette and supply bottle are connected 
at the top with a small wash-bottle, containing an alkaline pyrogallic acid solution, through which all the air entering, when some of the solution is withdrawn, has to pass and is deprived of most of its oxygen. The second neck carries the vessel for holding the water, usually a pear-shaped vessel of about $\frac{1}{4}$ litre capacity, with a stop cock below (a separator), by means of which the water can be run into the Woulf's bottle. The delivery tube from this vessel should be carried nearly to the bottom of the bottle. Through the india-rubber stopper of the third neck, three tubes are passed; one, resching nearly to the bottom, is connected with an apparatus for generating hydrogen; the second, also reaching nearly to the bottom, is used as a syphon for drawing off the contents of the Woulf's bottle without admission of air. The lower, outer end of this syphon tube can be closed by a pinchcock. The third tube passes only just through the stopper, and is connected, outside, with a wash-bottle, through which the hydrogen, passing through the whole apparatus, can escape, while at the same time access of air to the apparatus is cut off by the water contained in the bottle.*

An experiment is carried out as follows :-The two burettes having been filled, the Woulf's bottle is entirely filled with water, all communications with the air are cut off, the syphon is opened, and the hydrogen turned on. The hydrogen entering the bottle, presses the water out through the syphon, and by the time all the water is expelled, the bottle is filled with hydrogen; after this a gentle current of hydrogen is kept up, as long as the experiment lasts. The syphon is closed, and a small quantity of water is run into the bottle through the separator (20 to 30 c.c., or thereabouts). This water is slightly coloured by running in a small quantity of indigo, and the colour of this indigo cautiously discharged $k y$ the careful dropping in of hyposulphite solution. When this is accomplished, the contents of the pear-shaped vessel, which has meanwhile been filled with the water to be examined, are run into the bottle, and the blue colour thereby restored to the contents once more cautiously discharged. The amount of hyposulphite solution required for this is noted, the contents of the Woulf's bottle withdrawn by means of the syphon, and the same process repeated; the mean of the two experiments gives the amount of hyposulphite required by the water under examination. It now only remains to standardise the hyposulphite solution, which is accomplished by repeating the above process, with fully ærated water of known temperature, taking also the mean of the hyposulphite required in two experiments. From the respective quantities of hyposulphite required, the amount of oxygen contained in the water under examination is calculated. At first, I assumed that he proportions of oxygen dissolved in the two waters stood in the same relation as the amount of hyposulphite solutions required to discharge the blue colour in the two, but experiment showed that this was not so. The amount of indigo added to the water is, after its reduction, as a rule, entirely insufficient to take up the whole of the oxygen dissolved in the quantity of water taken for an experiment, and this necessitates a correction. On carrying out the experiment, as described above, it will be noticed that when the blue colour has been discharged, by the cautious addition of hyposulphite, it will very speedily return, and when again discharged, will return again, and this will

* The apparatus may be obtained at Messrs. Townsend and Mercer, Bishopsgate-street Without. 
continue until twice the amount of hyposulphite required to bring about the first decolouration has been added, after which the blue colour does not reappear, if the apparatus contains hydrogen only. This same amount of hyposulphite is required, if in. stead of taking a small amount of indigo merely, an amount is taken sufficient, after reductions, to take up the whole of the oxygen in the quantity of water used. This proves that the action of the dissolved oxygen on the reduced indigo is not the same as, at any rate, the immediate action taking place between the dissolved oxygen and the hyposulphite, but that in the first case twice the amount of oxygen is taken up at once than in the latter. The amounts of hyposulphite solution required to discharge the blue colour for the first time have therefore to be corrected, according to the amount of indigo employed, before they fairly represent the relative proportions of oxygen contained in the tro waters compared. This correction is one balf the amount of hyposulphite solution required simply to reduce the indigo added, so long as this is insufficient to take up all the oxygen present in the water, and to divide the amount of hyposulphite required by two whenever the indigo used was sufficient, or more than sufficient, to take up the whole of the oxygen of the water. The following table, giving one series of experiments out of many, will render the point clearer :-

TABLE I.

$\begin{array}{cc}\begin{array}{r}\text { Amount of } \\ \text { water taken. }\end{array} & \begin{array}{r}\text { Amount of } \\ \text { indigo taken. }\end{array} \\ 50 \text { c.c. } & 4 \text { drops* } \\ 50,, & 8, \\ 50,, & 12, \\ 50,, & 60, \\ 100,, & 12,\end{array}$

Amount of
hyposulphite
required.
0.65 c.c.
$0.70,$,
0.75,
$1.25, "$
$135,$,

$\begin{gathered}\text { Corrected amount proportional } \\ \text { to the amount of oxygen in } \\ \text { the water. } \\ 0.60 \text { c.c. }=0.65-0.05 \\ 0.60,,=0.70-0.10 \\ 0.60,=0.75-0.15 \\ 0.62,=1.25-0.20 \\ 0.60,=1.35-0.15\end{gathered}$

When the water to be examined is fully rerated, this correction has no influence on the result; its influence is but slight if the water is near its full æration, but it becomes very noticeable if the water falls considerably short of full æration. This correction would be unnecessary if the amount of indigo taken were always sufficient to take up, after reduction, the whole of the oxygen present in the water, but in fully arated water this amount is so great that it is difficult to fix the point at which the last trace of blue has been discharged with any degree of accuracy, since the solution never becomes colourless, but remains more or less yellow. I, therefore, prefer to use much less indigo and to take the point of first disappearance of the blue colour, afterwards applying the correction as above described. The amount of indigo I generally talse is such that for its decolouration or reduction about $1 / 10$ the amount of hyposulphite will be required than that used in the examination of a water fully ærated at mean temperature.

The actual amount of oxygen contained in the water used for standardizing, is calculated by means of Bunsen's formula, but in order to save trouble, Mr. Dibdin has calculated the amount contained in a gallon of water at various temperatures, so that it can at once be read off.

* 4 drops indigo required 0.1 cc. hyposulphite for their decolouration, corrections to be applied, therefore $0.05 \mathrm{cc}$. for every 4 drops indigo taken. the water.

In this case more indigo was taken than could be oxidised, after its reduction, by the oxygen of 


\section{I'ABLE II.*}

'lable shewing the quantity of Oxygen dissolved by water at various temperatures. Corrected to $0^{\circ} \mathrm{C}$. at $760^{\mathrm{m} . \mathrm{m} .}$ pressure.

\begin{tabular}{|c|c|c|c|c|c|c|}
\hline $\begin{array}{l}\text { Temperatur } \\
\text { Fahrenheit }\end{array}$ & & & $\begin{array}{l}\text { mperatur } \\
\text { entigrade }\end{array}$ & & & $\begin{array}{l}\text { Cubic Inches } \\
\text { of Oxygen } \\
\text { per Gallon. }\end{array}$ \\
\hline $41^{\circ}$ & on & .. & $5 \cdot 00$ & $\cdots$ & . & $2 \cdot 101$ \\
\hline 42 & . & . & $5 \cdot 55$ & $\ldots$ & .. & 2.074 \\
\hline 43 & .. & $\ldots$ & $6 \cdot 11$ & . & . & $2 \cdot 048$ \\
\hline 44 & . & .. & $6 \cdot 66$ & $\ldots$ & $\ldots$ & 2.022 \\
\hline 45 & . & . & $7 \cdot 22$ & $\ldots$ & .. & $1 \cdot 997$ \\
\hline 46 & . & . & $7 \cdot 77$ & . & .. & 1.973 \\
\hline 47 & .. & .. & $8 \cdot 33$ & $\cdots$ & . & $1 \cdot 949$ \\
\hline 48 & .. & . & $8 \cdot 89$ & .. & .. & $1 \cdot 927$ \\
\hline 49 & . & . & $9 \cdot 44$ & .. & . & 1.905 \\
\hline 50 & . & $\ldots$ & $10 \cdot 00$ & $\ldots$ & $\ldots$ & 1.884 \\
\hline 51 & . & . & $10 \cdot 55$ & .. & . & $1 \cdot 864$ \\
\hline 52 & . & .. & $11 \cdot 11$ & $\ldots$ & .. & $1 \cdot 844$ \\
\hline 53 & . & .. & $11 \cdot 66$ & .. & . & $1 \cdot 826$ \\
\hline 54 & $\ldots$ & $\ldots$ & $12 \cdot 22$ & $\ldots$ & $\ldots$ & $1 \cdot 808$ \\
\hline 55 & . & $\cdots$ & $12 \cdot 77$ & . & . & $1 \cdot 791$ \\
\hline 56 & . & .. & $13 \cdot 33$ & .. & .. & $1 \cdot 775$ \\
\hline 57 & $\ldots$ & .. & $13 \cdot 89$ & .. & .. & $1 \cdot 760$ \\
\hline 58 & . & .. & $14 \cdot 44$ & $\ldots$ & $\ldots$ & $1 \cdot 746$ \\
\hline 59 & .. & . & $15 \cdot 00$ & . & & $1 \cdot 732$ \\
\hline 60 & $\ldots$ & $\ldots$ & $15 \cdot 55$ & .. & . & $1 \cdot 719$ \\
\hline 61 & $\cdots$ & .. & $16 \cdot 11$ & $\ldots$ & . & $1 \cdot 706$ \\
\hline 62 & . & $\ldots$ & $16 \cdot 66$ & . & . & $1 \cdot 695$ \\
\hline 63 & $\ldots$ & . & $17 \cdot 22$ & .. & .. & $1 \cdot 683$ \\
\hline 64 & .. & $\cdots$ & $17 \cdot 77$ & . & . & $1 \cdot 674$ \\
\hline 65 & $\cdots$ & . & $18 \cdot 33$ & $\ldots$ & $\ldots$ & $1 \cdot 667$ \\
\hline 66 & . &. & $18 \cdot 89$ & .. & $\ldots$ & $1 \cdot 660$ \\
\hline 67 & .. & $\ldots$ & $19 \cdot 44$ & . & $\ldots$ & $1 \cdot 652$ \\
\hline 68 & . & .. & $20 \cdot 00$ & . & .. & $1 \cdot 644 \dagger$ \\
\hline 69 & & & 20.55 & .. & . & $1 \cdot 639^{\prime}$ \\
\hline 70 & .. & .. & $21 \cdot 11$ & .. & $\ldots$ & $1 \cdot 634$ \\
\hline
\end{tabular}

The result of the analysis may be given in different forms. Either the amount of oxygen found in the sample under examination may be given in cubic inches per gallon, or, as I prefer to do, in per cent. of possible amount, or in both. Thus, say a water, having a temperature of $20^{\circ} \mathrm{C}$, required 4 c.c. hyposulphite, the fully ærated water, at the same temperature, requiring 6 c.c. of the same solution, while the amount of indigo used took 0.6 c.c. of the same hyposulphite for its reduction. The corrected amounts of hyposulphite are $4-0.3=3.7$ and $6-0.3=5.7$. From Table II. we learn that a fully ærated water at $20^{\circ} \mathrm{C}$., holds in solution 1.644 cubic inch oxygen per gallon, and we get the proportion $5 \cdot 7: 1 \cdot 644=3 \cdot 7: x$.

$$
\mathrm{x} .=1 \cdot 067 \text {. }
$$

$$
\text { or, 2nd } 5 \cdot 7: 100:: 3 \cdot 7: x \text {; } x .=64 \cdot 9 \text {. }
$$

that is, the water contained 1.067 cubic inches of oxygen dissolved per gallon, and its degree of xration was 64.9 per cent. of the total possible.

* The table is calculated for a barometer pressure of $760 \mathrm{m.m}$. and would require corrections for variations from this, but such correction is mostly within the limits of experimental error.

+1.644 cubic inch of oxygen at $0^{\circ} \mathrm{C}$, and $760 \mathrm{~m} . \mathrm{m}$. pressure, weigh 0.594 grains. 
One final precaution, sometimes required, should be mentioned. In every case the water to be examined, must, of course, be transferred to the measuring vessel in a manner to avoid æration. This can generally be done by introducing it by means of a syphon passing to the bottom of the vessel, and thus filling it from below. In case, however, the water is but very partially ærated, it will absorb oxygen even while thus filling it into and running it out from the measuring vessel. In order to avoid this, I fill the vessel, in such cases, with carbonic acid, previous to putting in the water, and allow carbonic acid only to enter as the water runs out again. The effect of this is well shown in the following experiment:-A water which, by boiling, had been deprived of most of its dissolved oxygen required, when tested as usual, 0.83 c.c. hyposulphite, whereas, when carbonic acid was used as above directed, it required only 0.15 c.c. of the same; fully ærated water required 10.3 c.c. hyposulphite and the amount of indigo taken required 0.8 c.c. hypo. According to the first experiment, the degree of wration of the boiled water was 4.34 per cent., according to the second, 0.75 per cent. only.

It only remains to describe the preparation of the hyposulphite solution. A concentrated solution of bisulphite of sodium is saturated with sulphurous acid, a bottle of a fow ounces capacity is nearly filled with this solution, a small quantity of zinc dust is added, the bottle is closed and shaken for about ten minutes; should it become warm, it must be cooled-easiest under a running tap. The bottle is then allowed to rest, and after the excess of zinc has settled, the clear solution is decanted into a second bottle, of about ten times the capacity of the first. In this bottle thin milk of lime is added to alkaline reaction, after which, the bottle is completely filled with water, stoppered, shaken, and left at rest. The clear liquid, after the precipitate has settled, is the hyposulphite solution required. If too strong, it can easily be reduced by further dilution with pure water. The strength I prefer is such, that $\frac{1}{4}$ litre of fully arated water requires about 5 c.c. of the hypo. solution. It may, however, be used both stronger and considerably weaker.

In conclusion, I will give the degrees of æration found at various points of the river Thames on two days (Aug. 9 and Sept. 2, 1882), a comparison between which and the amount of oxygen absorbed from permanganate, as given in my paper printed in the July number of the ANaLYsT, will be found interesting. Temperature of the water Aug. $9,65.5^{\circ}$ F. ; on Sept. $2,60.5^{\circ}$ F.

Locality.

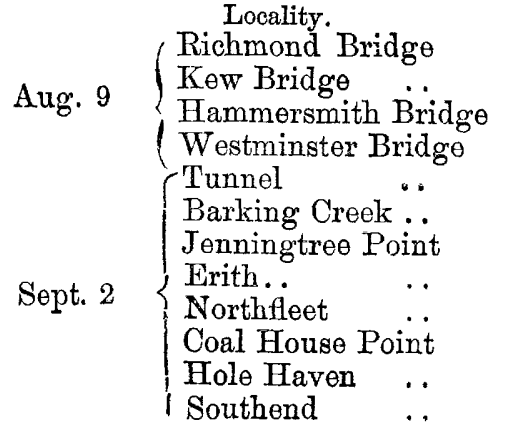

Percentage of Alration.

$\begin{array}{ll}\ldots & 100 \\ \ldots & 100 \\ . & 67 \cdot 3 \\ \ldots & 54 \cdot 5 \\ \ldots & 25 \\ \ldots & 19 \cdot 7 \\ \ldots & 23 \cdot 3 \\ \ldots & 23 \cdot 7 \\ \ldots & 38 \cdot 1 \\ \ldots & 52 \cdot 6 \\ \ldots & 75 \\ . & 96\end{array}$


It will be seen that the river starts fully ærated at Kew, becomes gradually deprived of its oxygen as it passes the metropolis, and finally re-acquires its proper amount. At first I was under the impression that even at Southend the river had not fully recovered its aration; but further experiments proved that this apparent deficiency was due to the fact that sea water, such as the water at Southend was, and usually is, at least at high water, is not capable of absorbing so much oxygen as river water, and that, in fact, the 96 per cent. found, by comparison with river water, represents fully ærated sea water.

Much more might be said regarding this process, and its applicability to water analysis, in which I am at present engaged; but I have already detained you too long, and can only express a final hope that other members of our Society will take up this extremely interesting subject, and bring their experience before us. 